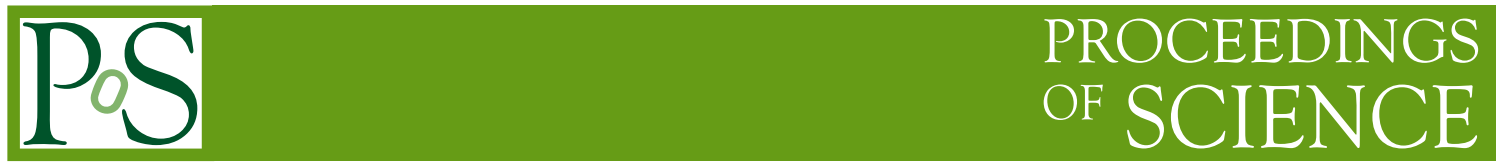

\title{
Studies of excited charm and beauty mesons at $\mathrm{LHCb}$
}

\section{Vladimir GLIGOROV*广}

European Organization for Nuclear Research (CERN), Geneva, Switzerland

E-mail: Vladimir.Gligorovdcern.ch

The LHCb detector is a general purpose forward spectrometer at the Large Hadron Collider, which exploits the $\approx 300 \mu \mathrm{b}$ cross-section for $b \bar{b}$ production and $\approx 6 \mathrm{mb}$ cross-section for $c \bar{c}$ production in $7 \mathrm{TeV}$ proton-proton collisions to make precise measurements of the properties of heavy flavour hadrons. Studies of excited $B$ and $D$ mesons using a dataset corresponding to $1 \mathrm{fb}^{-1}$ of integrated luminosity are presented. Measurements of masses, widths, and branching fractions are reported, as well as the first observation of the decay $B_{s 2}^{*}(5840)^{0} \rightarrow B^{*+} K^{-}$. This decay mode is then exploited in order to perform the world's most precise measurement of the $B^{*}-B$ mass difference.

XXI International Workshop on Deep-Inelastic Scattering and Related Subjects 22-26 April, 2013

Marseilles, France

\footnotetext{
${ }^{*}$ Speaker.

† On behalf of the LHCb collaboration.
} 


\section{Introduction}

The LHCb detector [ [ ] at the Large Hadron Collider (LHC) is a single arm spectrometer optimized for the study of charm and beauty hadrons. The LHCb acceptance covers the pseudorapidity range $2<\eta<5$; in what follows "transverse" means transverse to the LHC beamline. The detector includes a high-precision tracking system, electromagnetic and hadronic calorimeters, two ring-imaging Cherenkov detectors for hadron identification, and muon chambers for muon identification. The combined tracking system has a momentum resolution $\Delta p / p$ that varies from $0.4 \%$ at $5 \mathrm{GeV} / c$ to $0.6 \%$ at $100 \mathrm{GeV} / c$, an impact parameter ${ }^{1}$ resolution of $20 \mu \mathrm{m}$ for tracks with high transverse momentum, and a decay time resolution of $50 \mathrm{fs}$. Events are selected for further offline analysis by a trigger [] system based around a multivariate [B] inclusive topological $b$-hadron selection and high purity exclusive $c$-hadron triggers.

The $\approx 300 \mu \mathrm{b}$ cross-section [田] for $b \bar{b}$ and $\approx 6 \mathrm{mb}$ cross-section [ [5] for $c \bar{c}$ production in $7 \mathrm{TeV}$ proton-proton collisions, and a trigger system able to efficiently distinguish both leptonic and hadronic $b$-hadron decays, as well as certain $c$-hadron topologies such as $D^{ \pm} \rightarrow K^{\mp} \pi^{ \pm} \pi^{ \pm}$and $D^{0} \rightarrow K^{+} \pi^{-}$, from light-quark backgrounds, gives LHCb unprecedented statistical reach in the study of excited $b$ and $c$ hadrons. Such studies provide a stringent test of Heavy Quark Effective Theory (HQET), which is an important tool in calculating expected Standard Model values of $b$ and $c$ hadron lifetimes, $C P$-violation, mixing, and other parameters sensitive to potential physics effects beyond the Standard Model. The results presented here are based on a dataset corresponding to $1 \mathrm{fb}^{-1}$ of integrated luminosity. Ground-state $b$ and $c$ hadrons are selected exploiting their large masses and lifetimes, as well as LHCb's particle identification systems, to separate them from the background. These are then combined with "companion" kaons and pions in order to form excited $b$ and $c$ hadron candidates. The excited hadron candidates are passed through additional selection algorithms in order to improve their signal purity, before being fitted to extract the physics parameters of interest. The excited $c$-hadron studies will be presented first, followed by the excited $b$-hadron studies.

\section{Excited $D_{S J}$ meson studies}

The first result which will be presented is the study of $D_{s J}$ meson decays into $D^{0} K^{+}$and $D^{+} K_{S}^{0}$ final states [ [6]. The $D^{0, \pm}$ meson candidates are preselected in the $D^{ \pm} \rightarrow K^{\mp} \pi^{ \pm} \pi^{ \pm}$and $D^{0} \rightarrow K^{+} \pi^{-}$ final states, and their resulting mass distributions are shown in Fig. 1 . Subsequently, companion $K^{+}$ and $K_{S}^{0}$ particles are added to the $D$ vertices to form $D_{S J}$ candidates. These are further purified using particle identification and transverse momentum requirements, as well as by exploiting the cosine of the angle $\theta$ between the companion track in the $D_{S J}$ candidate rest frame and the momentum of the $D_{S J}$ candidate in the lab frame. This variable is symmetrically distributed around zero for resonant states, but more than $90 \%$ of combinatorial background lie in the negative region; a cut of $\cos (\theta)>0$ is therefore applied.

The resulting $D_{S J}$ mass distribution is shown in Fig. $\square$ for both $D^{0} K^{+}$and $D^{+} K_{S}^{0}$ candidates. We find around 0.36 million $D^{+} K_{S}^{0}$ and 3.15 million $D^{0} K^{+}$candidates. The same pattern of

\footnotetext{
${ }^{1}$ Impact parameter is the distance of closest approach between a track and a vertex, most commonly the primary proton-proton interaction vertex.
} 

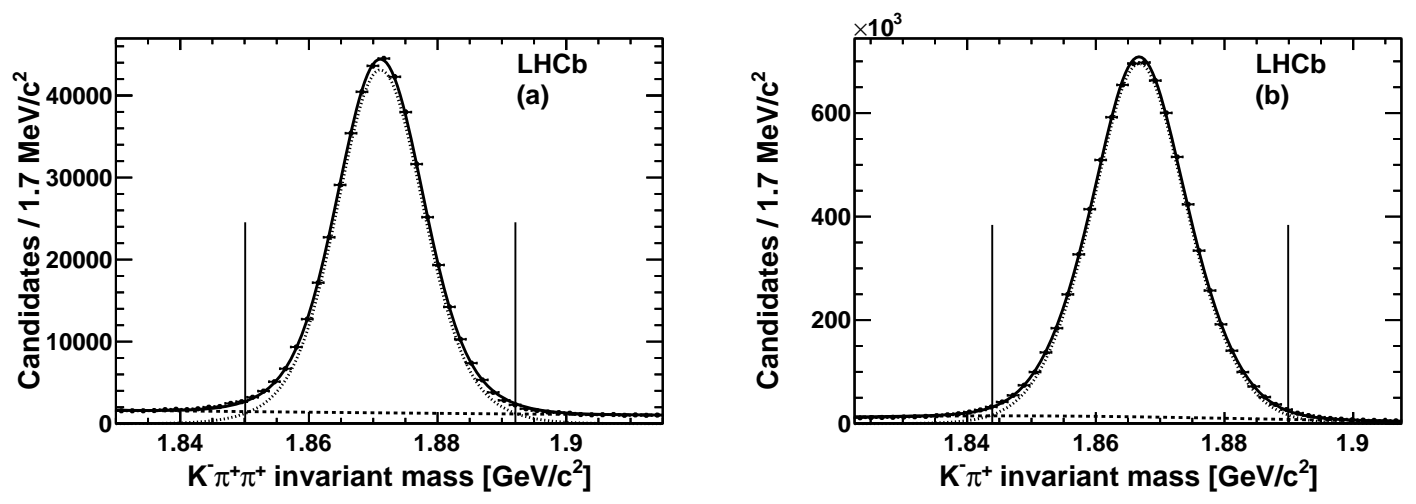

Figure 1: The (a) $D^{ \pm} \rightarrow K^{\mp} \pi^{ \pm} \pi^{ \pm}$and (b) $D^{0} \rightarrow K^{+} \pi^{-}$signals used in the $D_{S J}$ studies. The solid line is the total fit, the dashed line is the background. The vertical lines denote the signal region.
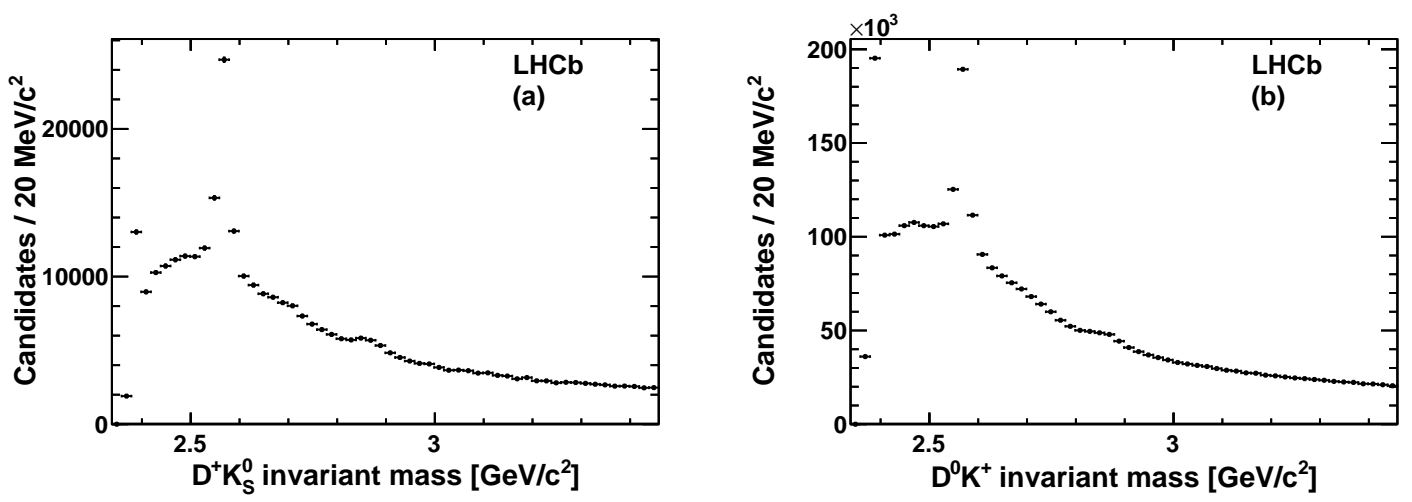

Figure 2: The $D_{S J}$ mass distributions for the (a) $D^{+} K_{S}^{0}$ and (b) $D^{0} K^{+}$combinations.

states is seen in both cases : a sharp, isolated, peak near the threshold due to feeddown from $D_{s 1}(2536)^{+} \rightarrow D^{*+} K_{S}^{0}, D^{* 0} K^{+}$decays, a large peak corresponding to the $D_{s 2}^{*}(2573)^{+}$spin-2 resonance, and two higher mass broad structures associated with the $D_{s 1}^{*}(2700)^{+}$and $D_{s J}^{*}(2860)^{+}$ states. A binned simultaneous maximum likelihood fit of these two distributions is used to measure the masses and widths of the higher mass broad resonances. Signals are modelled with Relativistic Breit-Wigner shapes including Blatt-Weisskopf form factors, and resolution effects are neglected in the nominal fit (but included as a systematic uncertainty) because the width of the states is between one and two orders of magnitude greater than the experimental resolution. The background distribution is dominated by randomly associated $D K$ pairs formed during hadronization and is described by a linear combination of Chebyshev polynomials of orders one to six. The nominal fit is shown in Fig. [3. The biggest systematic uncertainties are associated to the signal and background models, while smaller systematic uncertainties arise from the selection criteria, neglecting the mass resolution, and inclusion of possible additional feeddown shapes in the fit model. The final results 

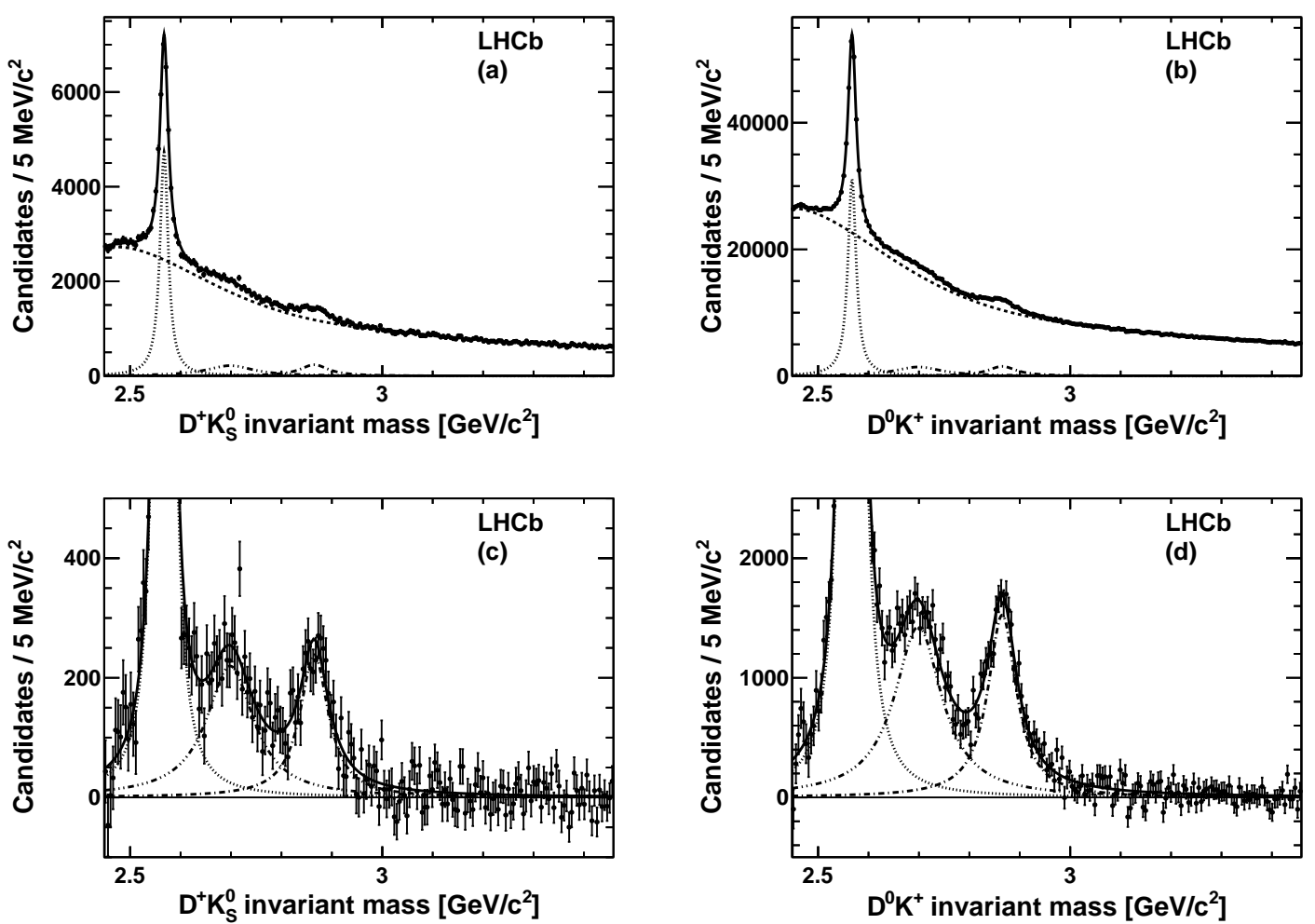

Figure 3: The fits to the $D_{s J}$ mass distributions for the $D^{0} K^{+}$and $D^{+} K_{S}^{0}$ combinations. The bottom plots show the background-subtracted mass distributions, zoomed around the modes of interest. The signals are, from left to right : the $D_{s 2}^{*}(2573)^{+}$, the $D_{s 1}^{*}(2700)^{+}$, and the $D_{s J}^{*}(2860)^{+}$. The solid line is the total fit, the dashed line is the background, while the dotted and dash-dotted lines are the signals.

are

$$
\begin{array}{lrl}
m\left(D_{s 1}^{*}(2700)^{+}\right)= & 2709.2 \pm 1.9 \text { (stat) } \pm 4.5 \text { (syst) } & \mathrm{MeV} / c^{2} \\
\Gamma\left(D_{s 1}^{*}(2700)^{+}\right)= & 115.8 \pm 7.3 \text { (stat) } \pm 12.1 \text { (syst) } & \mathrm{MeV} / c^{2} \\
m\left(D_{s J}^{*}(2860)^{+}\right)= & 2866.1 \pm 1.0 \text { (stat) } \pm 6.3 \text { (syst) } & \mathrm{MeV} / c^{2} \\
\Gamma\left(D_{s J}^{*}(2860)^{+}\right)= & 69.9 \pm 3.2 \text { (stat) } \pm 6.6 \text { (syst) } & \mathrm{MeV} / c^{2}
\end{array}
$$

where the uncertainties are statistical and systematic, respectively. The precision of the measurements is dominated by systematic effects and is comparable to previous BaBar [ $[0]$ and BELLE [ [ $]$ measurements.

\section{Excited $B_{s}^{0}$ meson studies}

The analysis of the excited $B_{s}^{0}$ meson states proceeds in a similar manner to the $D_{S J}$ analysis. First high-purity samples of fully reconstructed $B^{ \pm}$mesons are selected in four decay modes : $B^{+} \rightarrow J / \psi K^{+}, B^{+} \rightarrow \bar{D}^{0}\left(K^{+} \pi^{-}\right) \pi^{+}, B^{+} \rightarrow \bar{D}^{0}\left(K^{+} \pi^{-} \pi^{-} \pi^{+}\right) \pi^{+}$, and $B^{+} \rightarrow \bar{D}^{0}\left(K^{+} \pi^{-}\right) \pi^{+} \pi^{-} \pi^{+}$. 

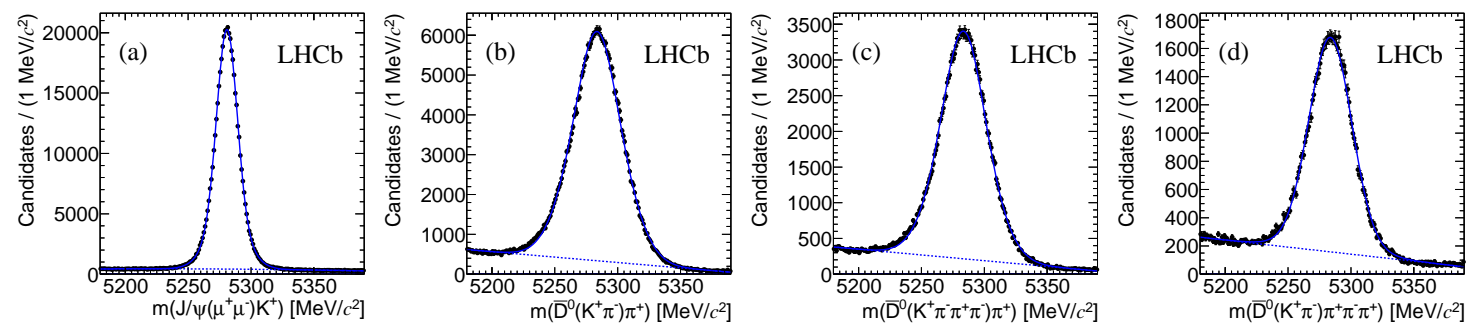

Figure 4: Invariant mass spectra of the final $B^{+}$candidates. The signal lineshape is fitted with a double Gaussian distribution, while the background is modelled with a second order polynomial . (a) $B^{+} \rightarrow J / \psi K^{+}$, (b) $B^{+} \rightarrow \bar{D}^{0}\left(K^{+} \pi^{-}\right) \pi^{+}$, (c) $B^{+} \rightarrow \bar{D}^{0}\left(K^{+} \pi^{-} \pi^{-} \pi^{+}\right) \pi^{+}$, and (d) $B^{+} \rightarrow \bar{D}^{0}\left(K^{+} \pi^{-}\right) \pi^{+} \pi^{-} \pi^{+}$decays. The $J / \psi$ and $D^{0}$ masses are constrained to their world average values.

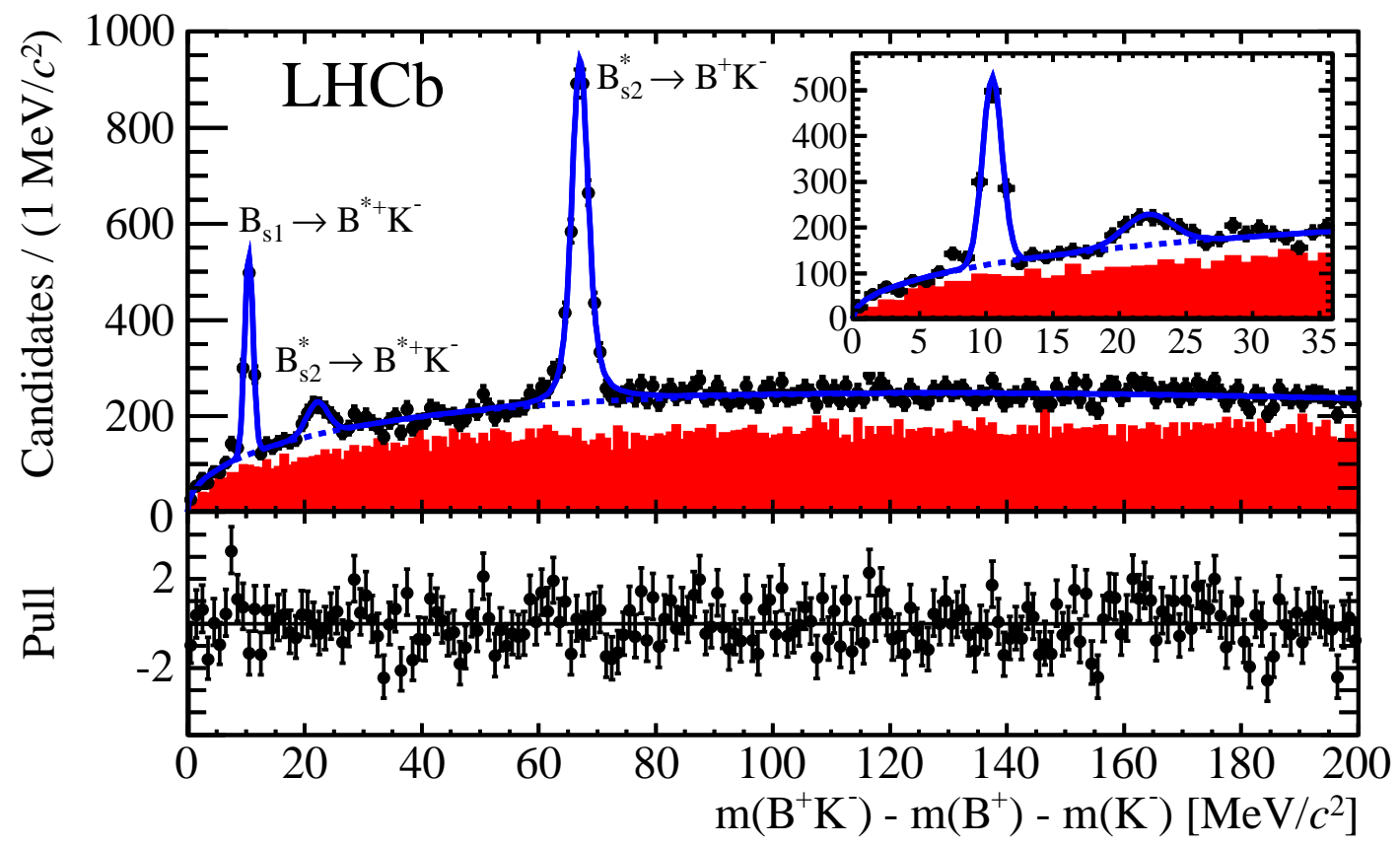

Figure 5: Mass difference distribution $m\left(B^{+} K^{-}\right)-m\left(B^{+}\right)-m\left(K^{-}\right)$. The three peaks are identified as (left) $B_{s 1} \rightarrow B^{*+} K^{-}$, (middle) $B_{s 2}^{*} \rightarrow B^{*+} K^{-}$, and (right) $B_{s 2}^{*} \rightarrow B^{+} K^{-}$. The total fit function is shown as a solid blue line, while the shaded red region is the spectrum of like-charge $B^{+} K^{+}$combinations. The inset shows an expanded view of the $B_{s 1} / B_{s 2}^{*} \rightarrow B^{*+} K^{-}$signals. The bottom plot shows the fit pulls.

The resulting $B^{+}$mass distributions are shown in Fig. Q $⿴$; around one million $B^{+}$candidates are obtained for further analysis.

Subsequently the $B^{* *}$ candidates are formed by combining the B candidate with a companion kaon. The $B^{* *}$ candidates are selected using a boosted decision tree algorithm which exploits transverse momenta, particle identification, and vertex quality information to discriminate signal from background. The obtained mass difference distribution $m\left(B^{+} K^{-}\right)-m\left(B^{+}\right)-m\left(K^{-}\right)$is shown in Fig. [1. The three peaks, left to right, are identified as $B_{s 1} \rightarrow B^{*+} K^{-}, B_{s 2}^{*} \rightarrow B^{*+} K^{-}$, and $B_{s 2}^{*} \rightarrow$ $B^{+} K^{-}$. In the case of the two leftmost peaks the decay contains a missing photon in the $B^{*+} \rightarrow$ $B \gamma$ transition. The fit to this mass distribution is performed using a threshold function for the background, a Relativistic Breit Wigner function convolved with a Gaussian to account for the 
Table 1: Results of the fit to the mass difference distributions $m\left(B^{+} K^{-}\right)-m\left(B^{+}\right)-m\left(K^{-}\right)$. The first uncertainties are statistical and the second are systematic.

\begin{tabular}{|c|c|c|}
\hline Parameter & Fit result & Best previous measurement \\
\hline$\overline{m\left(B_{s 1}\right)-m\left(B^{*+}\right)-m\left(K^{-}\right)}$ & $10.46 \pm 0.04 \pm 0.04 \mathrm{MeV} / c^{2}$ & $10.73 \pm 0.21 \pm 0.14 \mathrm{MeV} / c^{2}[9]$ \\
\hline$m\left(B_{s 2}^{*}\right)-m\left(B^{+}\right)-m\left(K^{-}\right)$ & $67.06 \pm 0.05 \pm 0.11 \mathrm{MeV} / c^{2}$ & $66.96 \pm 0.39 \pm 0.14 \mathrm{MeV} / c^{2}[\mathrm{Q}]$ \\
\hline$m\left(B^{*+}\right)-m\left(B^{+}\right)$ & $45.01 \pm 0.30 \pm 0.23 \mathrm{MeV} / c^{2}$ & $45.6 \pm 0.8 \quad \mathrm{MeV} / c^{2}$ [ए0] \\
\hline$\Gamma\left(B_{s 2}^{*}\right)$ & $1.56 \pm 0.13 \pm 0.47 \mathrm{MeV} / c^{2}$ & \\
\hline$\frac{\mathscr{B}\left(B_{s 2}^{*} \rightarrow B^{*+} K^{-}\right)}{\mathscr{B}\left(B_{s 2}^{*} \rightarrow B^{+} K^{-}\right)}$ & $(9.3 \pm 1.3 \pm 1.2) \%$ & \\
\hline$\frac{\sigma\left(p p \rightarrow B_{s 1} X\right) \mathscr{B}\left(B_{s 1} \rightarrow B^{*+} K^{-}\right)}{\sigma\left(p p \rightarrow B_{s 2}^{*} X\right) \mathscr{B}\left(B_{s 2}^{*} \rightarrow B^{+} K^{-}\right)}$ & $(23.2 \pm 1.4 \pm 1.3) \%$ & \\
\hline$N_{B_{s 1} \rightarrow B^{*+} K^{-}}$ & $750 \pm 36$ & \\
\hline$N_{B_{s 2}^{*} \rightarrow B^{*+} K^{-}}$ & $307 \pm 46$ & \\
\hline$N_{B_{s 2}^{*} \rightarrow B^{+} K^{-}}$ & $3140 \pm 100$ & \\
\hline
\end{tabular}

experimental resolution for the $B_{s 2}^{*} \rightarrow B^{+} K^{-}$signal, and two "effective" Gaussian functions to account for the other signals, where the missing photon causes a smearing in the signal shape which dominates the natural width. The results of the fit, and comparison to the best previous measurements, are shown in Tab. [1. The decay mode $B_{s 2}^{*} \rightarrow B^{*+} K^{-}$is observed for the first time and its branching fraction measured relative to that of $B_{s 2}^{*} \rightarrow B^{+} K^{-}$; furthermore, this signal is then used to make the most precise measurement to date of the $B^{*+}-B^{+}$mass difference. Because this measurement does not require the reconstruction of the soft photon from $B^{*+}$ decays, it has a significantly smaller systematic uncertainty than other measurements of this quantity.

\section{Conclusion}

Studies of excited $B$ and $D$ mesons using a dataset corresponding to $1 \mathrm{fb}^{-1}$ of integrated luminosity collected with the LHCb detector have been presented. The first observation of the decay $B_{s 2}^{*}(5840)^{0} \rightarrow B^{*+} K^{-}$has allowed LHCb to perform the world's most precise measurement of the $B^{*}-B$ mass difference, while the observations of the $D_{s 1}^{*}(2700)^{+}$and $D_{s J}^{*}(2860)^{+}$states made by BaBar and BELLE have been confirmed and their masses and widths are measured with a comparable precision to previous results.

\section{References}

[1] A. A. Alves et al. [ LHCb Collaboration ], JINST 3, (S08005)2008.

[2] R. Aaij et al. [ LHCb Collaboration ], arXiv 1211.3055, accepted in JINST.

[3] V. V. Gligorov and M. Williams, JINST 8, (P02013)2013.

[4] R. Aaij et al. [ LHCb Collaboration ], Phys. Lett. B 694, (209)2010.

[5] R. Aaij et al. [ LHCb Collaboration ], JHEP 1210, (151)2012

[6] R. Aaij et al. [ LHCb Collaboration ], JHEP 694, (209)2010. 
[7] B. Aubert et al. [ BaBar Collaboration ], Phys. Rev. D 80, (092003)2009.

[8] J. Brodzicka et al. [ BELLE Collaboration ], Phys. Rev. Lett. 100, (092001)2008.

[9] T. Aaltonen et al. [ CDF Collaboration ], Phys. Rev. Lett. 100, (082001)2008

[10] Q.W. Wu et al., Phys. Lett. B 273, (177-200)1991 